\title{
'|||||||||||||||||||||||||||||||||||||||||||||||||||||||||||||||||.
}

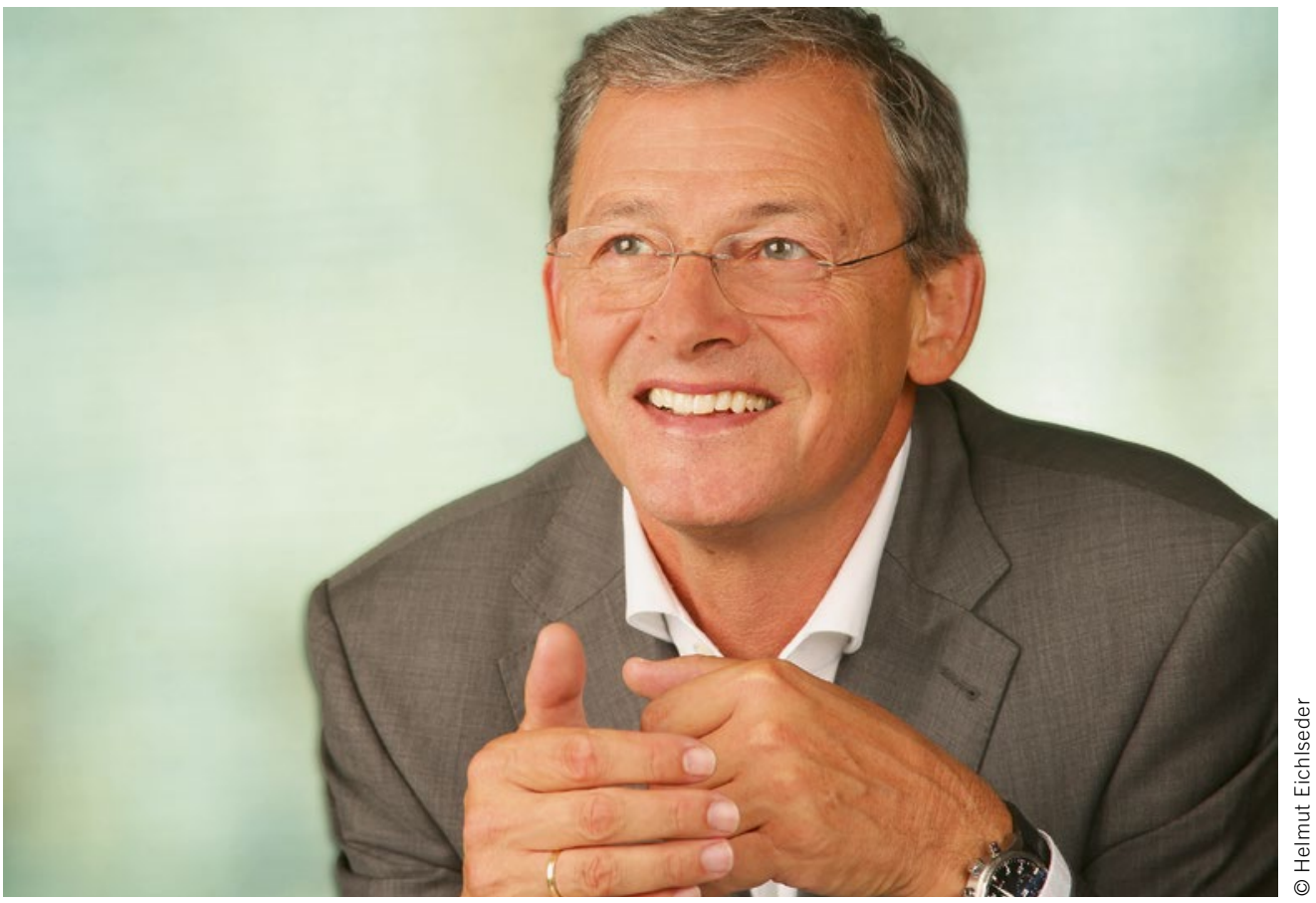

Univ. Prof. Dr. Helmut Eichlseder Vorstand des Instituts für Verbrennungskraftmaschinen und Thermodynamik (IVT) an der TU Graz

\section{Zero Impact - Eine klare Sache?}

In der jüngeren Vergangenheit wird häufig der Begriff „Zero Impact Vehicle“ (ZIV) verwendet, um die Umweltverträglichkeit zukünftiger Antriebssysteme zu artikulieren. Dass Zero Impact sowohl Klima- als auch Schadstoffrelevanz umfasst, wird zwar nicht immer differenziert, sollte heute aber zumindest weitgehend Konsens sein. Weit weniger klar ist die Definition des absoluten Niveaus an Schadstoffemissionen als auch klimarelevanter Effekte, um der Bezeichnung ZIV zu entsprechen.

Für eine exakte Definition bezüglich der Schadstoffemissionen gibt es eine Vielzahl möglicher Ansätze - abgesehen von der Sprachregelung „nicht mehr umweltrelevant“ oder „keine signifikanten Auswirkungen auf die Luftqualität“. Die Bandbreite reicht von ,gleichen Well-to-Wheel Emissionen wie ein ElektroPkw“ - die übrigens heute auf Basis Deutscher Stromerzeugung höher wären als die aktueller Euro-6-Pkw - bis zur „,NO $\mathrm{NO}_{2}$ Konzentration im Abgas kleiner als der Luftgütegrenzwert“. Eine plausible Definition ist für mich diejenige, dass der Beitrag von Pkw zu den Immissionen an hoch frequentierten Straßen in der Größenordnung sauberer, ländlicher Hintergrundwerte liegt, am Beispiel $\mathrm{NO}_{2}$ also bei etwa $3,5 \mu \mathrm{g} / \mathrm{m}^{3}$. Bereits heute unterschreiten mehrere Euro-6-Fahrzeuge die dementsprechenden Emissionswerte; mit der erwarteten Euro-7-Gesetzgebung ist für die Gesamtflotte von der Erfüllung obiger Zero Impact Definition auszugehen. Für die Definition der Grenzwerte sowie die Entwicklung und Anwendung neuer Emissionsminderungstechnologien sollte nicht die Frage nach technisch maxi- mal Machbarem entscheidend sein, sondern die nach den Erfordernissen der Luftqualität.

Wesentlich schwieriger ist die Anforderung an ZIV hinsichtlich der Klimarelevanz zu erfüllen; zumindest, wenn bezüglich $\mathrm{CO}_{2}$ nicht die aktuelle und technisch unrichtige Betrachtung Tank-to-Wheel, sondern eine des Gesamtsystems (Life-cycle Analysis, LCA) unterstellt wird. Selbst wenn nur die Energiebereitstellung berücksichtigt würde, kann Zero Impact hinsichtlich des Klimas nur auf Basis erneuerbarer Energie realisiert werden.

Dafür sind drei Hauptpfade möglich: elektrische Energie, Wasserstoff oder erneuerbare Kraftstoffe mit entsprechenden Energiewandlern beziehungsweise deren Kombination im Fahrzeug. Alle drei haben unterschiedliche Vor- und Nachteile, die sie für verschiedene Anwendungen prädestinieren. Eine „Universallösung“ existiert, entgegen einer weit verbreiteten Meinung, nicht.

Wichtige Kriterien neben der ausschließlich technischen Bewertung sind die Leistbarkeit für breite Bevölkerungsschichten, die Wertschöpfung innerhalb Europas und die (Rückwärts-) Kompatibilität mit bestehender Flotte und Infrastruktur. Nur damit kann über eine breite Anwendung in absehbarer Zeit der erforderliche, real wirksame Klimabeitrag geleistet werden.

Der Verbrennungsmotor hat jedenfalls bei einem Betrieb mit E-Fuels - zu denen auch Wasserstoff gehört - das Potenzial, die Kriterien eines Zero-Impact-Antriebes in allen relevanten Bereichen überzeugend zu erfüllen. 